\title{
The pronounced decline of anti-SARS-CoV-2 spike trimeric IgG and RBD IgG in baseline seronegative individuals 6 months after BNT162b2 vaccination is consistent with the need for vaccine boosters
}

\section{Gian Luca Salvagno}

Section of Clinical Biochemistry, University of Verona, Verona, Italy and Service of Laboratory Medicine, Pederzoli Hospital, Peschiera del Garda, Italy

\section{Brandon M. Henry}

Clinical Laboratory, Division of Nephrology and Hypertension, Cincinnati Children's Hospital Medical Center, Cincinnati, $\mathrm{OH}$, USA and Disease Intervention \& Prevention and Population Health Programs, Texas Biomedical Research Institute, San Antonio, Texas, USA

\section{Laura Pighi}

Section of Clinical Biochemistry, University of Verona, Verona, Italy and Service of Laboratory Medicine, Pederzoli Hospital, Peschiera del Garda, Italy

\section{Simone De Nitto}

Section of Clinical Biochemistry, University of Verona, Verona, Italy and Service of Laboratory Medicine, Pederzoli Hospital, Peschiera del Garda, Italy

\section{Gianluca Gianfilippi}

Medical Direction, Pederzoli Hospital, Peschiera del Garda, Italy

Giuseppe Lippi ( $\nabla$ giuseppe.lippi@univr.it)

Section of Clinical Biochemistry, University of Verona, Verona, Italy

\section{Short Report}

Keywords: COVID-19, SARS-CoV-2, Vaccination, Antibodies, Immune response

Posted Date: November 9th, 2021

DOI: https://doi.org/10.21203/rs.3.rs-1063499/v1

License: (c) (1) This work is licensed under a Creative Commons Attribution 4.0 International License. Read Full License 
The pronounced decline of anti-SARS-CoV-2 spike trimeric IgG and RBD IgG in baseline seronegative individuals 6 months after BNT162b2 vaccination is consistent with the need for vaccine boosters

\section{Gian Luca Salvagno $^{1,2}$, Brandon M. Henry ${ }^{3,4}$, Laura Pighi ${ }^{1,2}$, Simone De Nitto ${ }^{1,2}$, Gianluca Gianfilippi, ${ }^{5}$ Giuseppe Lippi ${ }^{1}$}

1. Section of Clinical Biochemistry, University of Verona, Verona, Italy

2. Service of Laboratory Medicine, Pederzoli Hospital, Peschiera del Garda, Italy

3. Clinical Laboratory, Division of Nephrology and Hypertension, Cincinnati Children's Hospital Medical Center, Cincinnati, OH, USA

4. Disease Intervention \& Prevention and Population Health Programs, Texas Biomedical Research Institute, San Antonio, Texas, USA

5. Medical Direction, Pederzoli Hospital, Peschiera del Garda, Italy

Short title: Anti-spike SARS-CoV-2 IgG antibodies after BNT162b2 vaccination

Word count: $1076+1$ Figure +1 Table

Corresponding author:

Prof. Giuseppe Lippi

Section of Clinical Biochemistry

University Hospital of Verona

Piazzale L.A. Scuro, 10

37134 Verona - Italy

Tel. 0039-045-8122970

Fax. 0039-045-8124308

Email: giuseppe.lippi@univr.it 


\begin{abstract}
Background: This observational retrospective study aimed to define the kinetics of serum levels of anti-SARS-CoV-2 (severe acute respiratory syndrome coronavirus 2) spike trimeric and anti-receptor binding domain (RBD) IgG antibodies up to 6 months after BNT162b2 vaccination.
\end{abstract}

Methods: The sample consisted of 86 SARS-CoV-2 baseline seronegative subjects (median age 45 years, IQR 31-53 years; $52.3 \%$ females) undergoing vaccination with Pfizer/BioNTech BNT162b2. Blood was drawn before receiving the first and the second vaccine dose, as well as 1, 3 and 6 months after the second vaccine dose. The serum levels of anti-SARS-CoV-2 spike trimeric IgG and RBD IgG antibodies were assayed.

Results: The peak of both antibodies types was reached 1 month after the second dose (2808 and $2163 \mathrm{BAU} / \mathrm{mL}$ for anti-SARS-CoV-2 spike trimeric IgG and RBD IgG), after which serum levels progressively declined, falling after 6 months to $486 \mathrm{BAU} / \mathrm{mL}$ and $167 \mathrm{BAU} / \mathrm{mL}$ for anti-SARS-CoV-2 spike trimeric IgG and RBD IgG, respectively. The median rate of 6-month decline was $85 \%$ and $93 \%$ for anti-SARS-CoV-2 spike trimeric IgG and RBD IgG, respectively. The rate of vaccine recipients with serum antibodies levels above the $80 \%$ threshold of vaccine efficacy declined from over $95 \%$ at the peak to $72 \%$ and $5 \%$ at 6 months for anti-SARS-CoV-2 spike trimeric IgG and RBD IgG, respectively.

Conclusions: The results of this retrospective observational study are consistent with the need for timely administration of vaccine boosters to prevent that humoral immunity will wane.

Keywords: COVID-19; SARS-CoV-2; Vaccination; Antibodies; Immune response 


\section{Introduction}

While novel effective therapies against coronavirus disease 2019 (COVID-19) are being developed and deployed, mass vaccination remains at the heart of national and international campaigns aimed at limiting the spread and the unfavourable consequences of the ongoing severe acute respiratory syndrome coronavirus 2 (SARS-CoV-2) pandemic [1]. Several types of COVID-19 vaccines have been developed and many others are currently undergoing clinical validation. Nonetheless, major hurdles remain concerning their production, allocation and widespread availability, especially in low resource settings, such that optimizing the administration of prime dose(s) and additional "boosters" remain a primary challenge [2].

Several months after the start of many nationwide immunization campaigns, convincing evidence suggests that the efficacy of COVID-19 vaccines for protecting against SARS-CoV-2 infection and the risk of developing symptomatic or severe COVID-19 illness appears to be progressively waning, thus resulting in a constantly increasing risk of SARS-CoV-2 breakthrough infections, as demonstrated by recent studies $[3,4]$. Such vulnerability has been also attributed to progressive decline of postvaccination anti-SARS-CoV-2 neutralizing antibodies, to levels that may be ineffective at limiting the viral infection and preventing its severe complications [5]. Therefore, we designed this observational retrospective study to define the kinetics of serum levels of anti-SARS-CoV-2 spike trimeric and anti-receptor binding domain (RBD) IgG antibodies up to 6 months after BNT162b2 vaccination.

\section{Materials and Methods}

The initial study population consisted of 100 consecutive healthcare workers of Peschiera del Garda hospital (Italy), who were SARS-CoV-2 seronegative at baseline, and underwent voluntary vaccination with the Pfizer/BioNTech BNT162b2 mRNA- 
based vaccine (Comirnaty, Pfizer Inc., New York, NY, US; two dose of $30 \mu \mathrm{g}, 3$ weeks apart). Blood was drawn before receiving the first and the second vaccine dose, as well as 1, 3 and 6 months after the second vaccine dose. The serum levels of anti-SARSCoV-2 spike trimeric IgG and RBD IgG antibodies were assayed with DiaSorin Trimeric spike IgG (DiaSorin, Saluggia, Italy) and MAGLUMI SARS-CoV-2 S-RBD IgG (New Industries Biomedical Engineering Co., Ltd [Snibe], Shenzhen, China), respectively. The analytical and clinical performance of these immunoassays have been previously described elsewhere [6,7]. Notably, accuracy and correlation with neutralization titers were as high as 0.953 for DiaSorin Trimeric spike $\operatorname{IgG}$ [6], and 0.712 for MAGLUMI SARS-CoV-2 S-RBD IgG [7], respectively. Test results were expressed as Binding Antibodies Units (BAU)/mL, reported as median and interquartile range (IQR), and compared with the thresholds of $80 \%$ vaccine efficacy against symptomatic SARS-CoV-2 infection, as estimated by Feng and colleagues (i.e., 264 BAU/mL for anti-spike and $506 \mathrm{BAU} / \mathrm{mL}$ for anti-RBD antibodies, respectively) [8]. All study subjects provided written informed consent for vaccination and undergoing anti-SARS-CoV-2 antibodies monitoring. This observation retrospective study was performed in accordance with the Helsinki Declaration and approved by the Ethics Committee of the Provinces of Verona and Rovigo (59COVIDCESC; November 3, 2021).

\section{Results}

The final sample consisted of 86 SARS-CoV-2 baseline seronegative subjects (median age 45 years, IQR 31-53 years; 45 (52.3\%) females), as 14 subjects were lost on follow-up. The kinetics of both anti-SARS-CoV-2 spike trimeric IgG and RBD IgG antibodies up to 6 months after BNT162b2 vaccination is shown in Figure 1. The peak of both antibodies types was reached 1 month after the second dose (median 2808 and 
IQR 1659-4075 BAU/mL for anti-SARS-CoV-2 spike trimeric IgG; median 2163 and IQR 1247-3283 BAU/mL for anti-SARS-CoV-2 RBD IgG), after which the serum levels tended to progressively decline, falling after 6 months from the second vaccine dose to $486 \mathrm{BAU} / \mathrm{mL}$ (IQR, 255-705 BAU/mL) for anti-SARS-CoV-2 spike trimeric IgG and 167 BAU/mL (IQR, 89-240 BAU/mL) for anti-SARS-CoV-2 RBD IgG, respectively. The median rate of 6-month decline of antibodies levels compared to the peak values was $85 \%$ (IQR, 80-89\%) for anti-SARS-CoV-2 spike trimeric IgG and 93\% (IQR, 89-95\%) for anti-SARS-CoV-2 RBD IgG, respectively. The rate of vaccine recipients displaying serum antibodies levels above the $80 \%$ threshold value of vaccine efficacy against symptomatic SARS-CoV-2 infection is reported in Table 1, showing that after a peak of positivity 1 months after the second dose (i.e., >95\%), this rate declined significantly at 6 months to $72 \%$ for anti-SARS-CoV-2 spike trimeric IgG and to $5 \%$ for anti-SARS-CoV-2 RBD IgG, respectively. A significant inverse Spearman's correlation was found between the age of vaccine recipients and the rate of 6-month decline of both anti-SARS-CoV-2 spike trimeric IgG ( $\mathrm{r}=-0.30 ; 95 \% \mathrm{CI},-0.48$ to -0.09 ; $\mathrm{p}=0.005)$ and anti-SARS-CoV-2 RBD $\operatorname{IgG}(\mathrm{r}=-0.32 ; 95 \% \mathrm{CI},-0.50$ to $-0.11 ; \mathrm{p}=0.003)$, whilst no association was found with sex for both antibodies types ( $>00.50$ for both).

\section{Discussion}

The results of our study demonstrate that the serum levels of both anti-SARSCoV-2 spike trimeric IgG and RBD IgG significantly declined 6 months after receiving the second BNT162b2 vaccine dose, with a trend of reduction more accentuated in younger subjects. Most importantly, a significant number of vaccine recipients displayed serum levels of these antibodies below the thresholds that are currently considered effective against symptomatic SARS-CoV-2 infection, with such rate of anti-SARS-CoV-2 RBD IgG exceeding 95\% of all subjects (Table 1). 
Recent evidence has been provided that a third dose of BNT162b2 vaccine efficiently elicits anti-SARS-CoV-2 antibodies levels without significant adverse events [9], such that some national and international healthcare organizations such as the US Advisory Committee on Immunization Practices are now officially endorsing booster doses of COVID-19 vaccines in selected categories of subjects at enhanced risk of developing severe SARS-CoV-2 infection [10]. This strategy is strongly supported by recent Israeli nationwide data [11], showing that the third vaccine dose reinforces protection against severe SARS-CoV-2 infection in people who had been vaccinated for over 6 months. Nonetheless, data demonstrates that given the important relationship between anti-SARS-CoV-2 antibody titer and protection, not all subjects would need a third vaccine dose at 6 months, especially those whose serum antibodies levels are still sufficiently high. Vaccine reactogenicity and the consequent risk of developing severe systemic reactions is magnified in patients with elevated serum anti-SARS-CoV-2 antibody levels [12], thus supporting the use of serologic testing before booster administration. Moreover, serologic monitoring could enable identification of those with lower antibody values, in whom prioritization of vaccine booster would be appropriate and justified.

In conclusion, the results of this retrospective observational study suggest that the anti-SARS-CoV-2 RBD IgG antibodies had decreased below a protective level in the vast majority of BNT162b2 vaccine recipients after 6 months. The rate of subjects with adequate levels of anti-SARS-CoV-2 spike trimeric IgG remained acceptable at 6 months post-vaccination (i.e., 72\%), though their progressive decline is consistent with the need for timely administration of vaccine boosters before humoral immunity will wane further. 
Acknowledgments: The authors are thankful to the entire staff of the Pederzoli Hospital of Peschiera del Garda (Verona, Italy) for accepting to participate to this study.

Research funding: None declared.

Author contributions: All authors have accepted responsibility for the entire content of this manuscript and approved its submission.

Competing interests: Authors state no conflict of interest.

Informed consent: Informed consent was obtained from all subjects included in this study.

Ethical approval: The study protocol (59COVIDCESC; November 3, 2021) was cleared by the Ethics Committee of the Provinces of Verona and Rovigo. All subjects were informed of the study and voluntarily agreed to participate, providing a written consent.

\section{References}

1. Callaway E. The race for coronavirus vaccines: a graphical guide. Nature 2020;580:576-7.

2. Callaway E. COVID vaccine boosters: the most important questions. Nature 2021;596:178-80.

3. Cohn BA, Cirillo PM, Murphy CC, Krigbaum NY, Wallace AW. SARS-CoV-2 vaccine protection and deaths among US veterans during 2021. Science. 2021 Nov 4:eabm0620. doi: 10.1126/science.abm0620. Epub ahead of print. 
4. Thomas SJ, Moreira ED Jr, Kitchin N, Absalon J, Gurtman A, Lockhart S, et al. Safety and Efficacy of the BNT162b2 mRNA Covid-19 Vaccine through 6 Months. N Engl J Med 2021;385:1761-73.

5. Klompas M. Understanding Breakthrough Infections Following mRNA SARSCoV-2 Vaccination. JAMA. 2021 Nov 4. doi: 10.1001/jama.2021.19063. Epub ahead of print.

6. Mahmoud SA, Ganesan S, Naik S, Bissar S, Zamel IA, Warren KN, Zaher WA, Khan G. Serological Assays for Assessing Postvaccination SARS-CoV-2 Antibody Response. Microbiol Spectr 2021;9:e0073321.

7. Padoan A, Bonfante F, Cosma C, Di Chiara C, Sciacovelli L, Pagliari M, et al. Analytical and clinical performances of a SARS-CoV-2 S-RBD IgG assay: comparison with neutralization titers. Clin Chem Lab Med 2021;59:1444-52.

8. Feng S, Phillips DJ, White T, Sayal H, Aley PK, Bibi S, et al. Correlates of protection against symptomatic and asymptomatic SARS-CoV-2 infection. Nat Med. 2021 Sep 29. doi: 10.1038/s41591-021-01540-1

9. Eliakim-Raz N, Leibovici-Weisman Y, Stemmer A, Ness A, Awwad M, Ghantous $\mathrm{N}$, et al. Antibody Titers Before and After a Third Dose of the SARS-CoV-2 BNT162b2 Vaccine in Adults Aged $\geq 60$ Years. JAMA. 2021 Nov 5. doi: 10.1001/jama.2021.19885. Epub ahead of print.

10. Mbaeyi S, Oliver SE, Collins JP, Godfrey M, Goswami ND, Hadler SC, et al. The Advisory Committee on Immunization Practices' Interim Recommendations for Additional Primary and Booster Doses of COVID-19 Vaccines - United States, 2021. MMWR Morb Mortal Wkly Rep 2021;70:1545-52.

11. Saciuk Y, Kertes J, Shamir Stein N, Ekka Zohar A. Effectiveness of a third dose of BNT162b2 mRNA vaccine. J Infect Dis. 2021 Nov 2:jiab556. doi: 10.1093/infdis/jiab556. Epub ahead of print. 
12. Krammer F, Srivastava K, Simon V. Robust spike antibody responses and increased reactogenicity in seropositive individuals after a single dose of SARS-CoV-2 mRNA $\quad$ vaccine. $\quad$ MedRxiv 2021.01.29.21250653; doi: https://doi.org/10.1101/2021.01.29.21250653. 
Figure 1. Kinetics of anti-SARS-CoV-2 spike trimeric RBD IgG and Anti-SARS-CoV2 RBD IgG serum levels in seronegative recipients of BNT162b2 mRNA-based vaccine.

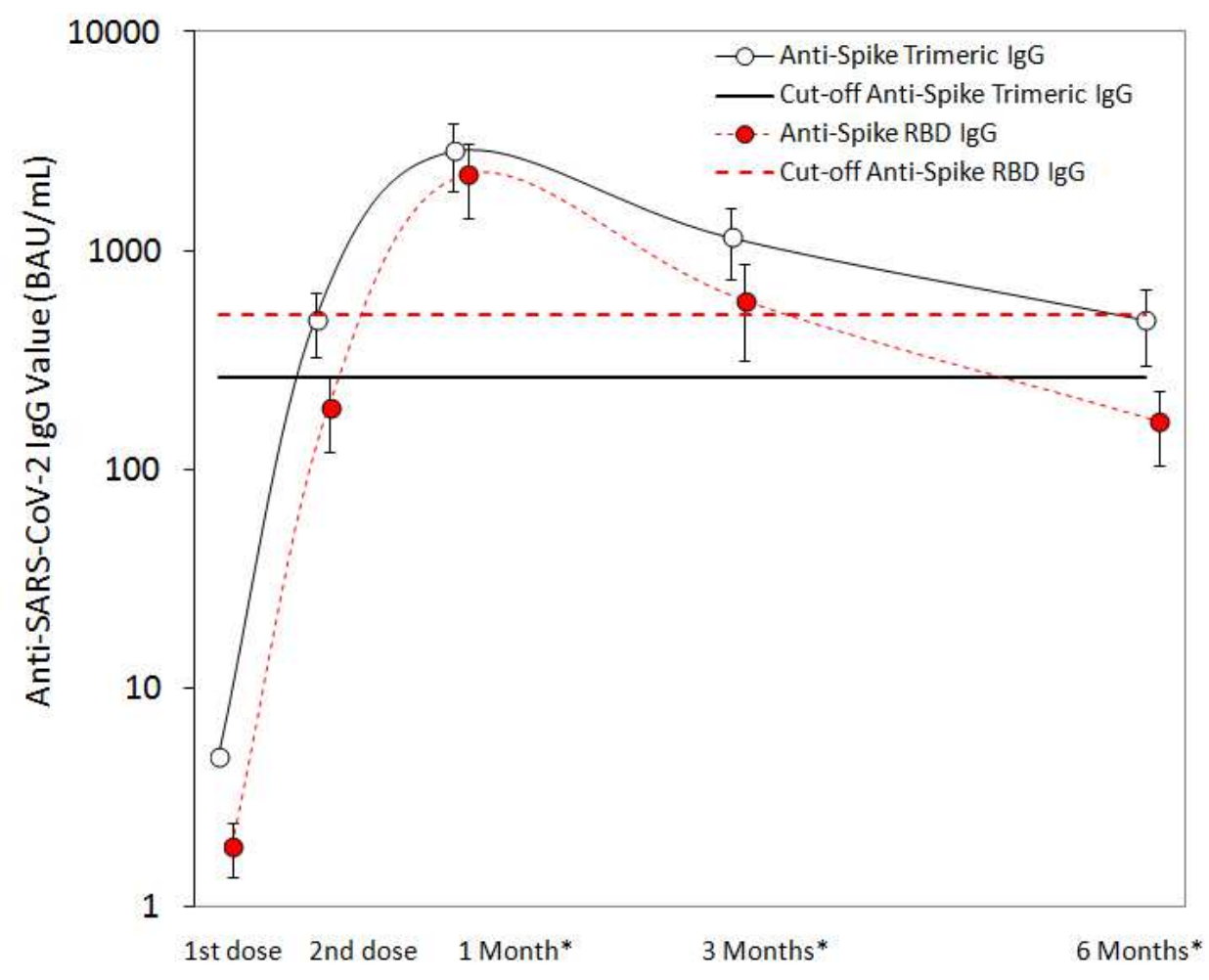

* After the second vaccine dose 
Table 1. Rate of vaccine recipients displaying serum antibodies levels above the $80 \%$ threshold value of vaccine efficacy against symptomatic SARS-CoV-2 infection.

\begin{tabular}{|l|c|c|c|c|c|}
\hline Antibody type & $\begin{array}{c}\text { Before 1 } \\
\text { dose }\end{array}$ & $\begin{array}{c}\text { Before 2 } \\
\text { dose }\end{array}$ & $\begin{array}{c}\mathbf{1} \\
\text { month* }^{*}\end{array}$ & $\begin{array}{c}\mathbf{3} \\
\text { months* }^{*}\end{array}$ & $\begin{array}{c}\mathbf{6} \\
\text { months* }^{*}\end{array}$ \\
\hline & & & & & \\
\hline $\begin{array}{l}\text { Anti-SARS-CoV-2 spike trimeric } \\
\text { RBD IgG (>264 BAU/mL) }\end{array}$ & $0 / 86$ & $66 / 86$ & $85 / 86$ & $81 / 86$ & $62 / 86$ \\
\hline & $0 \%$ & $77 \%$ & $99 \%$ & $94 \%$ & $72 \%$ \\
\hline $\begin{array}{l}\text { Anti-SARS-CoV-2 RBD IgG } \\
(>506 \text { BAU/mL) }\end{array}$ & $0 / 86$ & $7 / 86$ & $82 / 86$ & $41 / 86$ & $4 / 86$ \\
\cline { 2 - 6 } & $0 \%$ & $8 \%$ & $95 \%$ & $48 \%$ & $5 \%$ \\
\hline
\end{tabular}

* After the second vaccine dose 